\title{
Association Between Dental Caries and Body Mass Index in Schoolchildren Aged Between 14 and 16 Years in Riyadh, Saudi Arabia
}

\author{
Ahmed Abdullah Alghamdia , Ahmed Almahdyb,c
}

\begin{abstract}
Background: Dental caries and obesity are multifactorial diseases that have common risk factors. Studies worldwide reported varied outcomes about the association between dental caries and obesity. There is no published study that investigates this association among schoolchildren in Arabic countries. The aim of this study was to evaluate the relationship between dental caries (DMFT) and body mass index (BMI) for children aged between 14 and 16 years old in Riyadh, Saudi Arabia. The role of oral hygiene habits, parent's education, sugar consumption and physical activity in causing both diseases was also evaluated.
\end{abstract}

Methods: This is a cross-sectional study that involved 610 schoolchildren aged between 14 and 16 years. The children were selected from 12 boy's intermediate schools distributed in Riyadh city. A questionnaire that asked about demographic data, oral hygiene habits, parents' education, sugar consumption and physical activity was distributed. Then, the mean DMFT and BMI were calculated by collecting the information from dental examination, body weight and height. Multivariate logistic regression model was used to explain the relationship between the teeth health and the BMI. Moreover, multivariate linear regression was conducted to model the relationship between DMFT and BMI and the socioeconomic score, sugar consumption and physical activity.

Results: The prevalence of dental caries (DMFT > 0) was $54.1 \%$. Around $32 \%$ of the schoolchildren were either overweight or obese. A statistically significant association between dental caries (DMFT) and BMI was found $(\mathrm{P}=0.008)$. It was found that obese schoolchildren are 1.79 times higher to be with healthy teeth $(\mathrm{P}=0.02)$. In addition, it was found that schoolchildren with higher socioeconomic status are 1.26 times higher to be with healthy teeth group $(\mathrm{P}=0.005)$. Similarly, schoolchildren who reported that they are using the fluoridated

Manuscript submitted February 7, 2017, accepted March 1, 2017

${ }^{a}$ Ministry of Health, Abha, Saudi Arabia

bDepartment of Pediatric Dentistry and Orthodontics, College of Dentistry, King Saud University, Riyadh, Saudi Arabia

${ }^{\mathrm{c} C}$ Corresponding Author: Ahmed Almahdy, Department of Pediatric Dentistry and Orthodontics, College of Dentistry, King Saud University, Riyadh, Saudi Arabia.Email: a.almahdy@gmail.com

doi: https://doi.org/10.14740/jocmr2958w toothpaste were 1.63 times higher to be within the healthy teeth group $(\mathrm{DMFT}=0)$ when everything else is controlled. Physical activity level affects the schoolchildren BMI significantly $(\mathrm{P}<0.001)$.

Conclusions: The study showed a statistically significant association between dental caries and BMI for this study sample. Obese schoolchildren showed healthier teeth than others did. Fluoride toothpaste usage and socioeconomic score were associated significantly with dental caries.

Keywords: Dental caries; Body mass index; DMFT; Schoolchildren

\section{Introduction}

Dental caries is considered a multi-factorial lifestyle-related disease process involving the plaque biofilm which can be controlled by a combination of strategies addressing its etiological factors [1]. It is one of the most prevalent infectious diseases in the world and it is seven times more common than seasonal allergies [2]. Dental caries needs months or years to progress in most people. It is affected by consumption of dietary sugars, salivary flow, exposure to fluoride, and preventive procedures such as tooth brushing and regular check-ups [3]. Epidemiological studies reported increases in dental caries in both children and adults [4]. The prevalence of dental caries in Saudi Arabia was estimated to be approximately $70 \%$ for children's permanent dentition [5]. Al-Malik and Rehbini [6] showed that $96 \%$ of the children in their sample were diagnosed with dental caries and a high DMFT of 8.06 was scored. In addition, most of the Saudi children are considered to be at a high risk for developing dental caries [7].

According to WHO, obesity is defined as abnormal or excessive fat accumulation that may impair health. There is no cut-off point for excess fats of overweight or obesity in adolescents or children [8]. The fundamental principle that regulates gains or loss of weight is energy balance. In case of absorbing more energy than needed to physical activities and basal metabolism, the body will stock fat, and the opposite is also true [9]. There are multiple etiologies for this energy imbalance such as environmental factors, lifestyle, culture and behavior [8]. A recent study reported that the overweight prevalence among children in Riyadh was $19.5 \%$ in males and $20.8 \%$ in 
females [10]. Unfortunately, being overweight or obese aged 14 - 19 years was associated with increased mortality from systemic diseases [11].

In 2016, Alswat et al [12] found a significant positive correlation between BMI and DMFT among adults in Saudi Arabia. To our knowledge, there is no published study that assessed the association between dental caries and BMI among schoolchildren in Arabic countries.

The aim of this study was to evaluate the relationship between dental caries and BMI for schoolchildren aged between 14 and 16 years that are living in Riyadh, Saudi Arabia, with consideration of the roles of oral hygiene habits, parent's education, sugar consumption and physical activity.

\section{Materials and Methods}

This is a cross-sectional study, which was conducted in Riyadh, the capital of Saudi Arabia. The research proposal was reviewed and ethically approved by the institutional review board (IRB) in College of Medicine, King Saud University (no. 15/0521/IRB on December 24, 2015). Also, an approval from the Ministry of Education (no. 37661461) was obtained. The informed consent was distributed 1 week before the dental examination to the schoolchild's legal guardian and the approval was obtained prior to the examination.

A total of 610 schoolchildren aged between 14 and 16 years (third grade in Saudi intermediate school) were enrolled by multistage sampling technique. Fourteen educational sectors in Riyadh were reclassified according to their geographic locations into five sectors: eastern, southern, western, northern and central education sector. Then, a list of all intermediate boy's schools was obtained for each sector. After that, three schools were picked from the eastern and western sectors as they had large number of schools. Two schools were picked from the northern, southern and central sector. In each school, the participants were chosen randomly from a list of all schoolchildren aged between 14 and 16 years. The inclusion criteria were: age must be between 14 and 16 years, signed informed consent by schoolchild's legal guardian and the schoolchild must be present at the examination day. Schoolchildren who were not born and lived in Saudi Arabia were excluded.

Two pages' questionnaire with five sections obtained from WHO-oral health survey basic method (fifth edition, 2005) [13] was used with slight modifications. It contained demographic data, oral hygiene habits including tooth brushing frequency and fluoridated toothpaste usage, parents' education level, sugar consumption and physical activity. The questionnaire was filled by the schoolchildren on the same day prior to the dental examination and anthropometric measurement.

Dental examination was performed using sufficient quantity of disposable mouth mirrors, disposable dental probes and personal protective equipment (PPE). The used instruments were disposed safely after each examination to maintain adequate infection control in examination procedures. Each schoolchild was seated on a high-backrest chair and the examiner was standing in front of the chair. Information of decayed, missing teeth due to caries and filled teeth due to caries in per-
Table 1. Results From the Questioner $(n=610)$

\begin{tabular}{ll}
\hline Parental education & \\
Mother with higher education & $24.1 \%$ \\
Father with higher education & $34.7 \%$ \\
Tooth brushing habit & \\
Never & $11.9 \%$ \\
Several times a month & $23.0 \%$ \\
Several times a week & $46.3 \%$ \\
Daily & $18.9 \%$ \\
Use of flouridated toothpaste & \\
Do not know & $34.7 \%$ \\
No & $1.8 \%$ \\
Yes & $63.5 \%$ \\
Physical activity & \\
Never & $4.2 \%$ \\
Several times a month & $8.8 \%$ \\
Several times a week & $46.7 \%$ \\
Daily & $40.2 \%$ \\
\hline
\end{tabular}

manent dentition (DMFT) were obtained. A tooth was coded as a decayed when it has one of these conditions. 1) Lesion in a pit, fissure or on a smooth tooth surface that has an unmistakable cavity, undermined enamel or detectably softened wall or floor. 2) When the crown has been destroyed by caries and only the root is left. 3) Tooth with temporary or permanent restorations but decayed. Filled tooth was coded when one or more permanent restorations are present with no caries anywhere on the crown. The permanent tooth that has been extracted because of caries was coded as missed [13]. Any tooth being lost for other reasons, like trauma, was not documented.

Anthropometric measurement was done immediately after dental examination using digital plate scale to weigh the schoolchildren. The scale was on a firm surface and calibrated prior to each weight measurement. Schoolchildren stepped on the scale bare feet, without outer heavy cloths, empty pocket and facing away from the scale. Weight value was read to the nearest $100 \mathrm{~g}$ and recorded before the schoolchild got off the scale. Height was measured by measuring tape with strait, sided and relaxed arms and shoulders and with appropriate head position. The height was recorded to the nearest $1 \mathrm{~cm}$.

The principle investigator did both dental examination and anthropometric measurements for all schoolchildren. Intra-examiner validity was calculated by re-examining 30 schoolchildren in one randomly selected school.

Date was analyzed using SPSS-IBM version 20. Principal component analysis (PCA) was utilized to estimate composite scores for socioeconomic made from parental education and suburban location of residence. Standardized sugar consumption score was made from eight food items, which the schoolchildren were asked about. To understand the nature of relationship between dental caries and BMI and other predictors, a dichotomous variable was made $(\mathrm{DMFT}=0$ versus $\mathrm{DMFT}>$ 


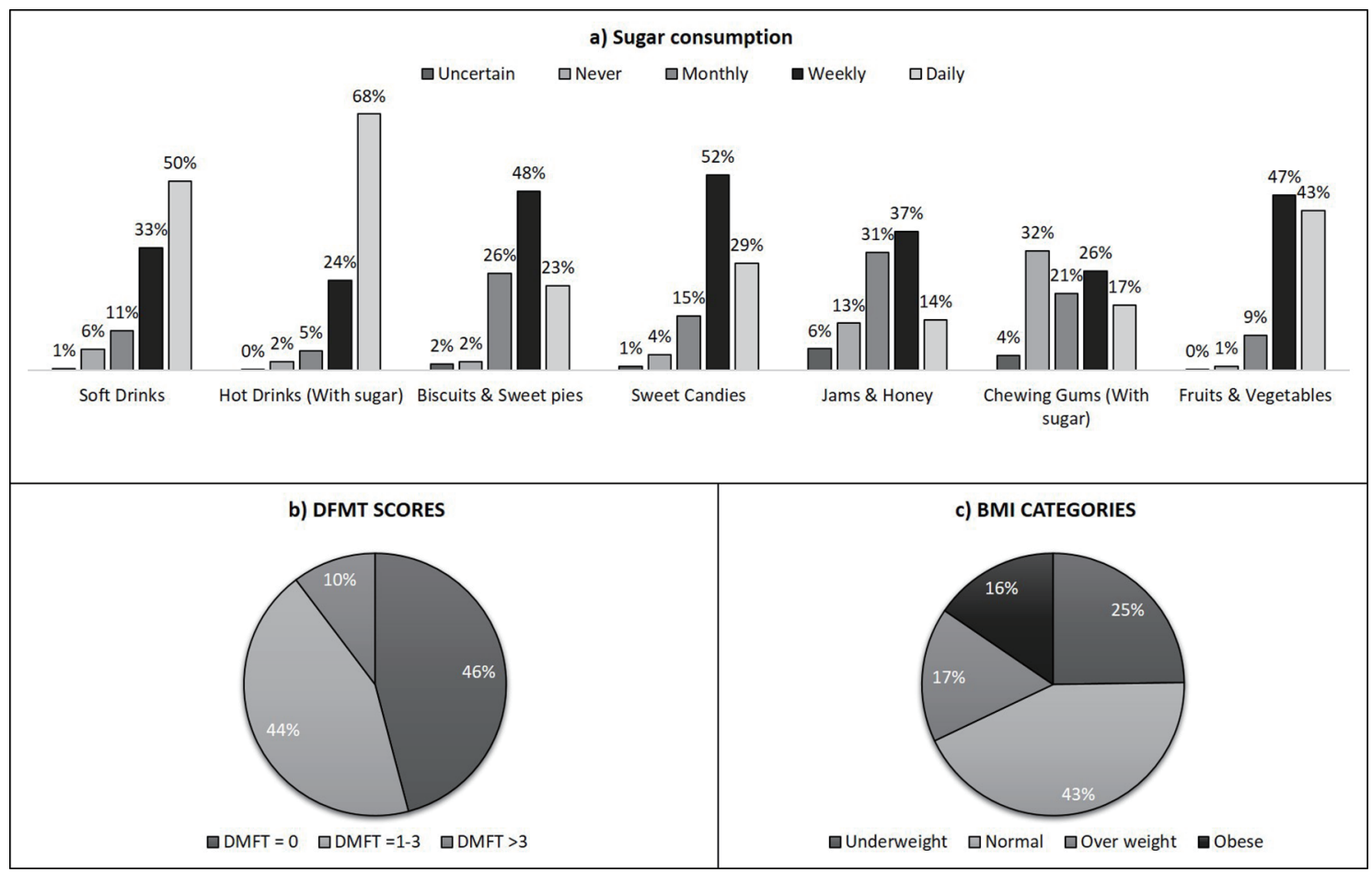

Figure 1. (a) Consumption of sugar-containing food percentages for all schoolchildren. (b) DMFT scores. (c) BMI categories (n $=610)$.

0) and multivariate logistic regression model was conducted. In addition, multivariate logistic regression model was used to explain the relationship between the DMFT and the rest of the predictors. Moreover, multivariate linear regression was conducted to model the relationship between BMI and socioeconomic score, sugar consumption and the other relevant predictors. The results were expressed as odds ratios (ORs) with 95\% confidence intervals (CIs) and alpha levels were set to 0.05 for all the tests throughout the analysis.

\section{Results}

Overall, 610 students were included in the study. All of them were males and they came from 12 schools located east, west, north, south and the city center of Riyadh, Saudi Arabia. The schoolchildren were from the same age group with minor variations in age. The youngest child was 14.1 years and the eldest was 15.9 years old. The results from the questionnaire are summarized in Table 1. The frequency of sugar-containing food consumption for the selected eight food items is shown in Figure 1a. In this sample, the prevalence of dental caries $($ DMFT $>0)$ was $54.1 \%$ (Fig. 1b). The majority of the schoolchildren $(43.2 \%)$ had a BMI falling within the normal expected range for this age group. Around $32 \%$ of the schoolchildren were either overweight or obese. The rest of the schoolchildren were underweight (24.8\%) (Fig. 1c). Intra-examiner validity was calculated and it was $89.2 \%$.

The multivariate logistic regression model showed that there is a statistically significant association between dental caries (DMFT) and BMI $(\mathrm{P}=0.008)$. As the BMI increases, the probability of having healthy teeth was increased (Fig. 2). It was found that obese schoolchildren are 1.79 times higher to be with healthy teeth $(\mathrm{DMFT}=0)$ when compared to those who had BMI within normal range $(\mathrm{P}=0.02)$.

In addition, it was found that schoolchildren with higher socioeconomic status are 1.26 times higher to be with healthy teeth group $(\mathrm{DMFT}=0)$ than those who are less $(\mathrm{P}=0.005)$ (Table 2). Similarly, schoolchildren who reported that they are using the fluoridated toothpaste were 1.63 times higher to be within the healthy teeth group (DMFT $=0$ ) when everything else is controlled (Table 2). On the other hand, children who consumed more sugar products (in the form of candies, biscuits, soft drinks, etc.) were predicted to have worse teeth $(\mathrm{DMFT}>0)$ when everything else is accounted for controlled (Table 2). The physical activity level was not significantly related to teeth health.

A multivariate linear regression model was conducted to understand how predictors like socioeconomic score, standardized sugar consumption and the physical activity level affect the schoolchildren's BMI. The later predicator showed significant negative relationship $(\mathrm{P}<0.001)$ with the schoolchildren's BMI when everything is accounted for. Both socioeconomic score and the standardized sugar consumption were not significantly correlated with the BMI of the schoolchildren.

\section{Discussion}

This research was conducted to evaluate the relationship be- 
Teeth situation for different BMI categories

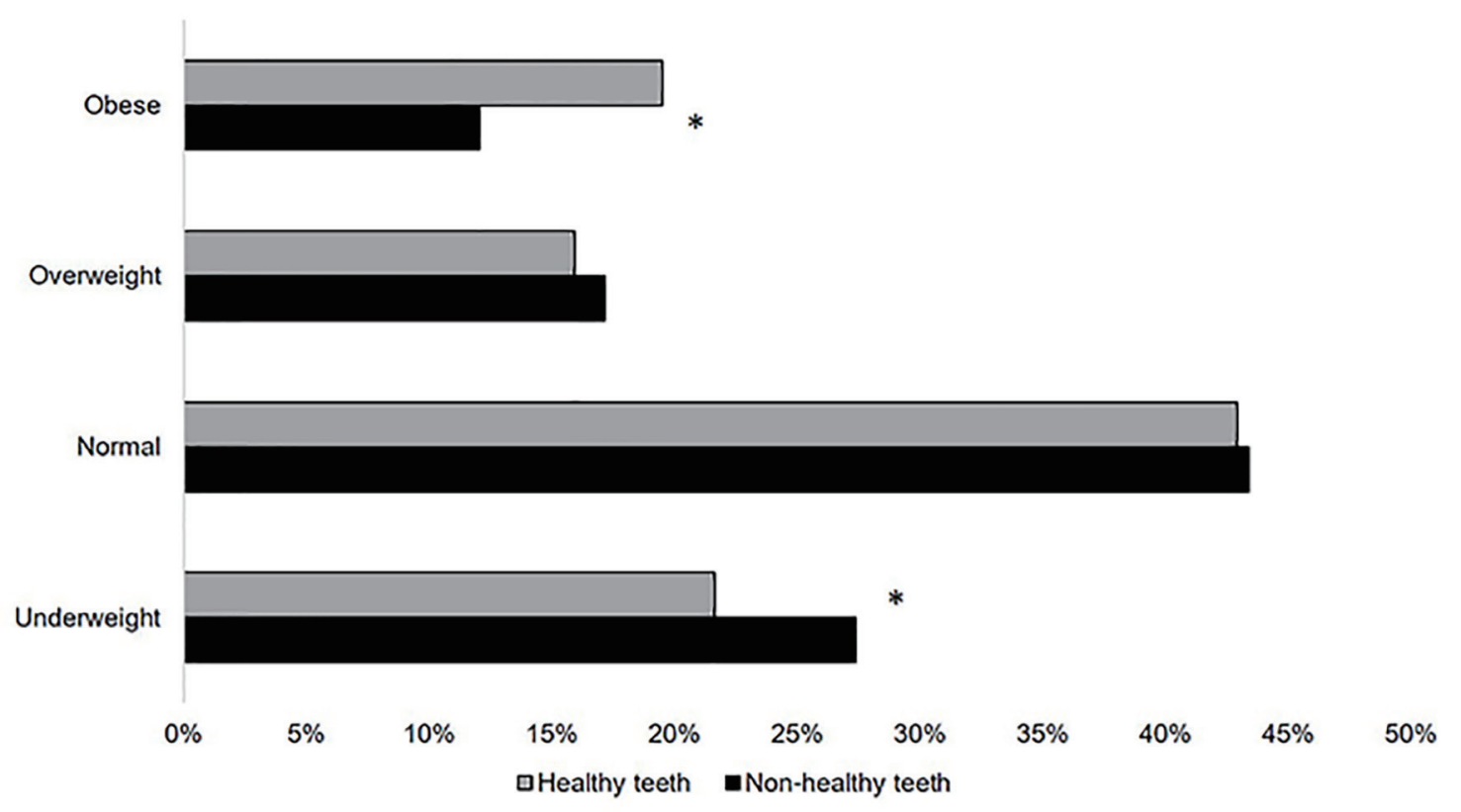

Figure 2. The percentage of having either a healthy tooth $(\mathrm{DMFT}=0)$ or non-healthy tooth $(\mathrm{DMFT}>0)$ for the different BMI categories $\left({ }^{*} \mathrm{P}<0.05\right)$.

tween dental caries and BMI for boys aged between 14 and 16 years who are born and live in Saudi Arabia. The roles of potential confounding factors including oral hygiene habits (brushing frequency and use of fluoride toothpaste), parents' education, diet and physical activity level were considered. The participants in this study were boys aged between 14 and 16 years. This is to avoid the effect of parental control, which is usually greater in early childhood, on the causative factors of both dental caries and obesity [14]. In addition, the presence of complete permanent dentition was insured. Only male schoolchildren were included to minimize the effect of gender variation. Several studies had demonstrated significant difference between gender [15, 16], age groups [17] and fluoride level among cities on dental caries prevalence [14]. In addition, the ability of doing physical activity of males in Saudi Arabia is much higher than the females.

A statistically significant negative association between dental caries and BMI was found in this study population. Obese schoolchildren, those with higher socioeconomic statues and who reported they used fluoridated toothpaste were more likely to be with healthy teeth $(\mathrm{DMFT}=0)$. Ideally, the schoolchildren must have normal BMI and healthy teeth. Many studies assessed the relationship between dental caries and BMI with varying outcomes. According to a systematic review, $48 \%$ of studies found no association between dental caries and BMI, 35\% found a positive association and 19\% found a negative association [17]. Beside the significant disagreement of the nature and existence of the association between dental caries and BMI in children, there are evidences supporting that the differences between samples such as the countries' level of development can associate with caries. Consequently, there is a negative association between dental caries and BMI reported from studies conducted in developing countries like Asia and South America. On the other hand, studies showing positive association were primarily conducted in Europe (Sweden, France and Germany) and the United States. No association was largely found in studies from United States, South America and Europe [17].

Many studies had reported that overweight children had lower dental caries when compared to normal weight children. In 2006, Macek and Mitola [18] found that the normal weight children have more dental caries experience in both primary

Table 2. Odd Ratios for Teeth Health Outcome as Measured by Healthy Teeth (1) and Non-Healthy Teeth (0) for Different Predictor

\begin{tabular}{|c|c|c|c|c|c|}
\hline & \multirow{2}{*}{ Odds ratio } & \multicolumn{2}{|c|}{$95 \%$ CI for OR } & \multirow{2}{*}{ Wald, $\mathbf{d f}=1$} & \multirow{2}{*}{ P value } \\
\hline & & Lower & Upper & & \\
\hline Socioeconomic score & 1.259 & 1.073 & 1.478 & 7.944 & 0.005 \\
\hline Standardized sugar consumption score & 0.867 & 0.736 & 1.022 & 2.894 & 0.089 \\
\hline The physical activity level & 1.419 & 0.859 & 2.345 & 1.870 & 0.171 \\
\hline
\end{tabular}


and permanent dentition. Similarly, Narksawat et al [19] found that overweight children were less likely to have dental caries in primary $(\mathrm{OR}=0.6)$ and permanent dentition $(\mathrm{OR}=0.5)$ than normal weight children. It was also found that underweight and normal weight schoolchildren were more likely to have dental caries at least 1.94 times and 2.22 times, respectively compared to overweight and obese children who are $12-14$ years old [19]. It had been suggested that underweight children eat more snacks that contain relatively high sugar, which increases the risk of developing dental caries, and make them miss or eat less amount of the main meals that are usually high in protein and fat when compared to overweight children [20]. This interpretation is consistent with this study finding. The underweight schoolchildren exhibited the highest sugar consumption, as well as the highest physical activity. Thus, they eat more sugar-containing foods that make them at higher risk of developing dental caries and they keep their weight by the high physical activity.

Caries prevalence (DMFT $>0$ ) was $54.1 \%$ with a mean DMFT of $1.26(\mathrm{SD}=1.56)$. This is considered low compared to the national prevalence of dental caries in the permanent dentition of Saudi children. The later was $70 \%$ with a mean DMFT of 3.5 [5]. However, it fit within the reported range for the children live in Riyadh, which was 52-93\% [5]. The low caries prevalence in the current study sample can be elucidated by several explanations. Firstly, a strict diagnostic method was used to determine the presence of sound, decayed, missing or filled tooth in this study. Secondly, the variation in dental examination methodology between surveys such as examination in the dental clinic or using dental radiograph, which can detect dental caries in its initial stages. Finally, the schoolchildren with the selected age group in this study had been recently transitioned from the primary dentition stage to the permanent dentition stage. This means that the most of the teeth were sound as they were newly erupted and there was no enough time to develop dental caries. Finally, it was pointed out in a meta-analysis of caries in the different regions of Saudi Arabia that the dental caries level in permanent teeth might be overestimated due to publication bias [21].

Many studies had shown that fluoride exposure led to slower progression in dental caries. It was found that children aged 5 - 16 years who used fluoridated toothpaste twice a day had fewer dental caries after 3 years regardless of whether they were drinking fluoridated water or using other fluoride products [22]. In this study sample, schoolchildren who reported they used the fluoridated toothpaste were 1.63 times higher to be within the healthy teeth group when compared to those who reported they do not or are uncertain. This also explained the low dental caries prevalence in our study sample.

Both the parental educational level and the residential area contributed in the socioeconomic score in this study. It was found schoolchildren with higher socioeconomic score are $26 \%$ more likely to have healthier teeth $(\mathrm{DMFT}=0)$ when everything else is accounted for. Similar finding was found by Al-Mohammadi et al [23] in 1997. They found that the dental caries prevalence increased among schoolchildren who belonged to low socioeconomic group in Riyadh city. In another study, an association between mother's education level and 6 years old children's dental caries level was found [24]. In the current study, no significant interaction between mother or father education with dental caries was found. This might be because the lesser effect of parental control with adolescence. In younger age children (probably less than 10 years) most of their life's aspects such as nutrition, hygiene and behaviors are more depending on parents. Many studies have included the family income in the evaluation of the socioeconomic score. However, this was not done in this study as the parent's education has more effect in Saudi Arabia the family income in relation to the awareness of both dental caries and obesity.

Future preventive measures should include preventive strategies to prevent dental caries and reduce risk of obesity in the community. The main effort should focus on changing bad dietary habits and promote physical activity and oral hygiene. Ministry of Education in Saudi Arabia made some strict rules for school cafeteria to prevent high sugar products. However, during school visits, we noticed that donuts and sweet chocolate bars still exist in school's cafeterias.

Prospective longitudinal studies are needed to investigate causal relationship between dental caries and obesity, and also to study the long-term association with more accurate indicators such as dietary patterns and health behaviors.

\section{Conclusion}

This study showed a statistically significant association between dental caries and BMI for the selected age in this study sample. Although it is not acceptable to have obesity among schoolchildren, the study showed that they have healthier teeth than others did. Fluoride toothpaste usage and socioeconomic score were associated significantly with dental caries. Teeth brushing frequency, parent's education, and physical activity were not statistically significant with dental caries.

\section{Acknowledgments}

Authors appreciated the support of ministry of education and schools' principals that facilitated study's procedures and help to accomplish on time. The authors also acknowledge the College of Dentistry Research Centre and Deanship of Scientific Research at King Saud University, Saudi Arabia for supporting the research.

\section{Grant Support}

Deanship of Scientific Research at King Saud University, Saudi Arabia.

\section{References}

1. Pitts NB, Wefel JS. Remineralization/desensitization: what is known? What is the future? Adv Dent Res. 2009;21(1):83-86.

2. Al-Darwish M, El Ansari W, Bener A. Prevalence of den- 
tal caries among 12-14 year old children in Qatar. Saudi Dent J. 2014;26(3):115-125.

3. Selwitz RH, Ismail AI, Pitts NB. Dental caries. Lancet. 2007;369(9555):51-59.

4. Bagramian RA, Garcia-Godoy F, Volpe AR. The global increase in dental caries. A pending public health crisis. Am J Dent. 2009;22(1):3-8.

5. Al Agili DE. A systematic review of population-based dental caries studies among children in Saudi Arabia. Saudi Dent J. 2013;25(1):3-11.

6. Al-Malik MI, Rehbini YA. Prevalence of dental caries, severity, and pattern in age 6 to 7-year-old children in a selected community in Saudi Arabia. J Contemp Dent Pract. 2006;7(2):46-54.

7. Farsi N, Merdad L, Mirdad S. Caries risk assessment in preschool children in Saudi Arabia. Oral Health Prev Dent. 2013;11(3):271-280.

8. Dehghan M, Akhtar-Danesh N, Merchant AT. Childhood obesity, prevalence and prevention. Nutr J. 2005;4:24.

9. Wright SM, Aronne LJ. Causes of obesity. Abdom Imaging. 2012;37(5):730-732.

10. El-Hazmi MA, Warsy AS. The prevalence of obesity and overweight in 1-18-year-old Saudi children. Ann Saudi Med. 2002;22(5-6):303-307.

11. Calcaterra V, Klersy C, Muratori T, Telli S, Caramagna C, Scaglia F, Cisternino M, et al. Prevalence of metabolic syndrome (MS) in children and adolescents with varying degrees of obesity. Clin Endocrinol (Oxf). 2008;68(6):868-872.

12. Alswat K, Mohamed WS, Wahab MA, Aboelil AA. The association between body mass index and dental caries: cross-sectional study. J Clin Med Res. 2016;8(2):147152.

13. Petersen PE, Baez RJ. World Health Organization, Oral health surveys: basic method. World Health Organization. 2013.

14. Silva AE, Menezes AM, Demarco FF, Vargas-Ferreira F, Peres MA. Obesity and dental caries: systematic review.
Rev Saude Publica. 2013;47(4):799-812.

15. Denloye O, Popoola B, Ifesanya J. Association between dental caries and body mass index in 12-15 year old private school children in Ibadan. Nigeria. Pediatric Dental Journal. 2015.

16. Begum $\mathrm{MZ}$, et al. Is body mass index truly related to dental caries? Survey on predisposing factors for overweight among Indian school children. Dental Hypotheses. 2014;5(4):150.

17. Hooley M, Skouteris H, Boganin C, Satur J, Kilpatrick N. Body mass index and dental caries in children and adolescents: a systematic review of literature published 2004 to 2011. Syst Rev. 2012;1:57.

18. Macek MD, Mitola DJ. Exploring the association between overweight and dental caries among US children. Pediatr Dent. 2006;28(4):375-380.

19. Narksawat K, Tonmukayakul U, Boonthum A. Association between nutritional status and dental caries in permanent dentition among primary schoolchildren aged 1214 years, Thailand. Southeast Asian J Trop Med Public Health. 2009; 40(2):338-344.

20. Summerbell CD, Moody RC, Shanks J, Stock MJ, Geissler C. Sources of energy from meals versus snacks in 220 people in four age groups. Eur J Clin Nutr. 1995;49(1):33-41.

21. Khan SQ, Khan NB, Arrejaie AS. Dental caries. A meta analysis on a Saudi population. Saudi Med J. 2013;34(7):744-749.

22. Marinho VC, Higgins JP, Logan S, Sheiham A. Fluoride gels for preventing dental caries in children and adolescents. Cochrane Database Syst Rev. 2002;2:CD002280.

23. al-Mohammadi SM, Rugg-Gunn AJ, Butler TJ. Caries prevalence in boys aged 2, 4 and 6 years according to socio-economic status in Riyadh, Saudi Arabia. Community Dent Oral Epidemiol. 1997;25(2):184-186.

24. Al-Sadhan SA. Oral health practices and dietary habits of intermediate school children in Riyadh, Saudi Arabia. 2003. 